\title{
Diagnostic accuracy of SPECT/CT arthrography in patients with suspected aseptic joint prostheses loosening
}

\author{
Bo Bao ${ }^{1 *}$ (D, Crystal S. Liu' ${ }^{2}$ Edward C. O. Masson ${ }^{3}$ and Jonathan T. Abele
}

\author{
*Correspondence: bbao@ualberta. \\ ca \\ ${ }^{1}$ Department of Radiology and \\ Diagnostic Imaging, University of \\ Alberta, 2A2.41 Walter C Mackenzie \\ Health Sciences Centre, Edmonton, \\ Alberta T6G 2B7, Canada \\ Full list of author information is \\ available at the end of the article
}

\begin{abstract}
Purpose: To evaluate the diagnostic accuracy of SPECT/CT arthrography in patients with suspected aseptic prosthesis loosening following hip and knee arthroplasty.

Methods: A retrospective review of 63 SPECT/CT arthrogram studies (36 knees and 27 hips) between February 1, 2013, and July 1, 2018, was conducted. All patients underwent clinical and radiologic evaluation as part of their assessment for persistent pain following hip and knee arthroplasty. The detection of tracer activity along the bone-prosthetic interface on SPECT/CT suggests aseptic loosening. Operative assessment as well as clinical/radiologic follow-up at a minimum of 1 year was used as the reference standard.
\end{abstract}

Results: The sensitivity and specificity of SPECT/CT for detection of aseptic loosening was 6/7 (86\%) and 55/56 (98\%), respectively. This gives a positive predictive value (PPV) of $6 / 7(86 \%)$, a negative predictive value (NPV) of 55/56 (98\%), and a diagnostic accuracy of 61/63 (97\%).

Conclusion: SPECT/CT arthrography has a high diagnostic accuracy (97\%) in the evaluation of loosening of both hip and knee arthroplasties in patients with persistent post-procedural pain.

Keywords: Total knee arthroplasty, Total hip arthroplasty, SPECT/CT, Loosening

\section{Springer Open}

\section{Introduction}

Hip and knee arthroplasties are commonly performed procedures for the management of osteoarthritis (Katz et al. 2010; Ethgen et al. 2004). In the USA, over 7.2 million individuals have received hip and knee replacement surgeries (Maradit Kremers et al. 2015). The rate of joint replacement surgery is projected to increase further in the next few years (Singh et al. 2019). While hip and knee arthroplasties are successful in most patients, persistent pain is a common complication that affects up to $44 \%$ of patients with total hip arthroplasty and 27\% of patients with total knee arthroplasty (Wylde et al. 2011; Piscitelli et al. 2013; Becker et al. 2011). Although post-surgical pain can be due to a variety of causes, aseptic loosening is the most common complication of hip and knee arthroplasty requiring major revision surgery (Sharkey et al. 2014). Hence,

(c) The Author(s). 2021 Open Access This article is licensed under a Creative Commons Attribution 4.0 International License, which permits use, sharing, adaptation, distribution and reproduction in any medium or format, as long as you give appropriate credit to the original author(s) and the source, provide a link to the Creative Commons licence, and indicate if changes were made. The images or other third party material in this article are included in the article's Creative Commons licence, unless indicated otherwise in a credit line to the material. If material is not included in the article's Creative Commons licence and your intended use is not permitted by statutory regulation or exceeds the permitted use, you will need to obtain permission directly from the copyright holder. To view a copy of this licence, visit http://creativecommons.org/licenses/by/4.0/. 
accurate detection of aseptic loosening is essential in guiding management decisions for persistent post-arthroplasty pain.

Aseptic loosening describes a failure in the integration of the bone and prosthesis that is not due to an infection (Sundfeldt et al. 2006). Instead, wear debris forms at the implant-bone interface and causes chronic inflammation. The end result is an osteolytic process that leads to loosening (Abu-Amer et al. 2007). Since progression of periprosthetic tissue destruction can be subtle, clinical diagnosis of aseptic loosening in patients is challenging. Initial workup typically involves a detailed history, clinical examination, and conventional radiographs during follow-up. For complex patients, adjunct imaging modalities such as bone scintigraphy, radionuclide arthrography, single photonemission computed tomography/computed tomography (SPECT/CT), 18F-FDG-PET, and/or MRI can be used for detecting aseptic loosening (Lohmann et al. 2017). In this retrospective study, our goal was to confirm the accuracy of SPECT/CT arthrography in patients with clinically suspected hip or knee arthroplasty loosening.

\section{Materials and methods}

\section{Data collection}

This study is a retrospective analysis of all SPECT/CT arthrogram studies referred to our center between February 1, 2013, and July 1, 2018. During this period, a total of 94 SPECT/CT arthrogram studies were performed for the evaluation of persistent pain after hip and knee arthroplasty. Institutional ethics review board approval of our study protocol was obtained.

Demographic information (age, gender), type of prosthesis (cemented vs noncemented), radiographic diagnosis, clinical findings, and surgical reports were exported for analysis. All patients had an initial standard radiograph exam followed by SPECT/ CT arthrogram as part of the diagnostic pathway. Patients were only included in the study if they were subsequently followed up by an orthopedic surgeon, had an operative revision, or a minimum of 1-year period of observation. As well, joint aspiration results during the follow-up period and after revision surgery were reviewed to exclude septic etiology. Six studies were excluded from analysis due to failed tracer injections as indicated by tracer activity seen outside the joint capsule on the SPECT/CT images (i.e., extracapsular injection despite fluoroscopic guidance). These patients were typically rebooked for a repeat study and the data from these repeats may be included in the data set. Twenty-five patients were excluded since no reference standard was available, either because they did not have subsequent operative assessment or no follow-up clinic data could be obtained. The remaining 63 patient studies were included for analysis (Fig. 1).

\section{Imaging procedure}

A 22-gage needle was inserted into the joint space of interest under fluoroscopic guidance by an experienced interventional radiologist. This was approached via the inferolateral margin of femoral neck component for those with a prior hip arthroplasty or subpatellar joint space for those with a prior knee arthroplasty. Positioning within the joint space was confirmed with the injection of $2 \mathrm{~mL}$ of iodinated contrast (Omnipaque 300, GE Healthcare, Buckinghamshire, UK). Once confirmed, $1 \mathrm{mCi}(37 \mathrm{MBq})$ of Tc- 


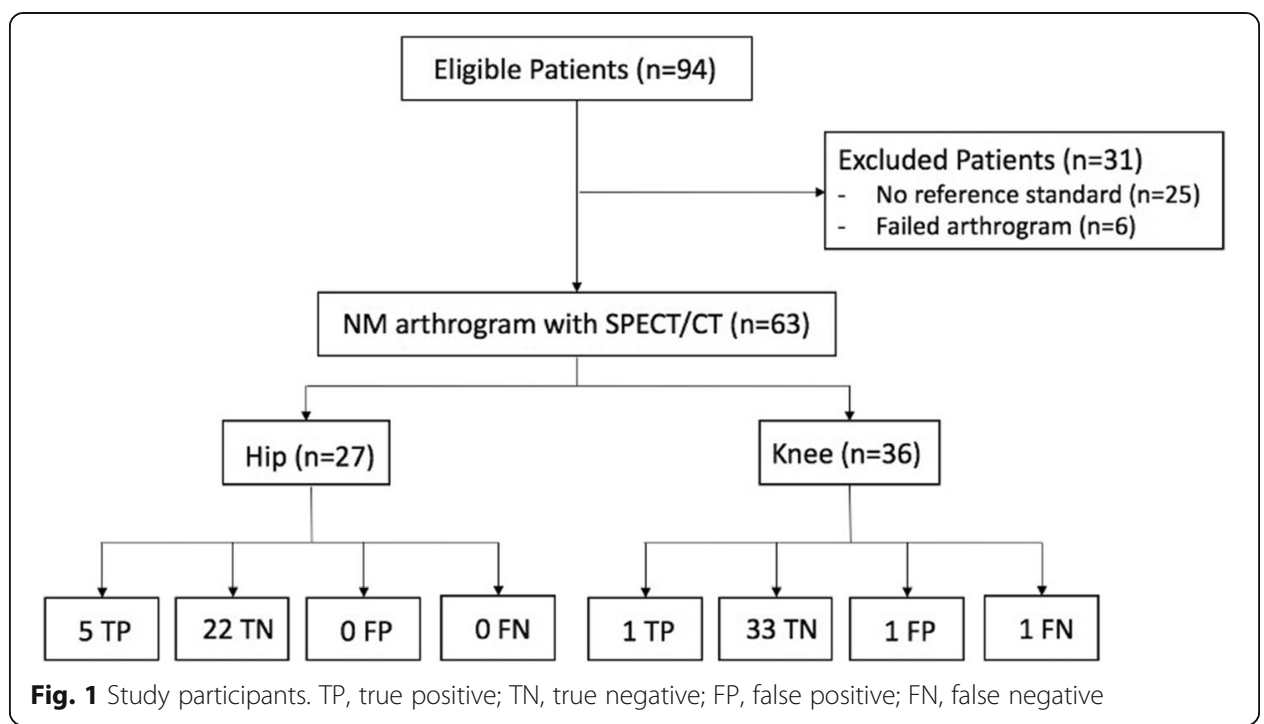

$99 \mathrm{~m}$ sulfur colloid in $2 \mathrm{~mL}$ of sterile saline was injected into the joint. The patient was then encouraged to ambulate for a minimum of $30 \mathrm{~min}$ before being imaged. If unable to ambulate, a minimum of 1 -h delay was required before imaging.

Initially, planar images were obtained in anterior, posterior, and lateral positions (128 $\times 128$ matrix, low-energy high-resolution collimator, $10 \mathrm{~min}$ per acquisition). SPECT/ CT images were then acquired using a hybrid SPECT/CT system equipped with a lowenergy, high-resolution collimator (Philips Precedence, Best, the Netherlands; Philips Brightview, Best, the Netherlands; or Siemens Symbia, Munich, Germany). The CT parameters were $80 \mathrm{~mA}, 120-130 \mathrm{kV}, 512 \times 512$ matrix size, and 1-5-mm slice thickness. SPECT/CT was performed with a matrix size of $128 \times 128,1.0$ zoom, $20 \mathrm{~s}$ per frame, and 120 frames at $3^{\circ}$ intervals.

All images were reconstructed using vendor-recommended iterative reconstruction algorithms. Decay correction and attenuation correction were both applied. No postreconstruction filter was applied.

All SPECT/CT arthrogram studies were reported by specialists licensed in nuclear medicine who were not blinded to previous imaging and clinical history. The SPECT/ CT images were reviewed using Oasis workstations (Segami Corporation, Columbia, $\mathrm{MD)}$. SPECT/CT studies were considered negative if tracer activity was only visualized within the joint space (Fig. 2) and positive for loosening if any tracer activity was seen along the bone-prosthetic interface outside of the joint space (Figs. 3 and 4). The study was considered a failed exam if an extracapsular injection was identified on the SPEC $\mathrm{T} / \mathrm{CT}$ images (these patients were typically re-booked for a repeat study).

\section{Reference standard}

Patients with persistent pain following primary arthroplasty were considered to have a loosened prosthesis if it was verified at the time of operative revision or if the patient had continued pain at 1-year follow-up with progressive radiographic features of loosening. The patients were determined not to have aseptic loosening if there was no evidence of loosening at time of operative assessment, if the pain resolved during 1-year 


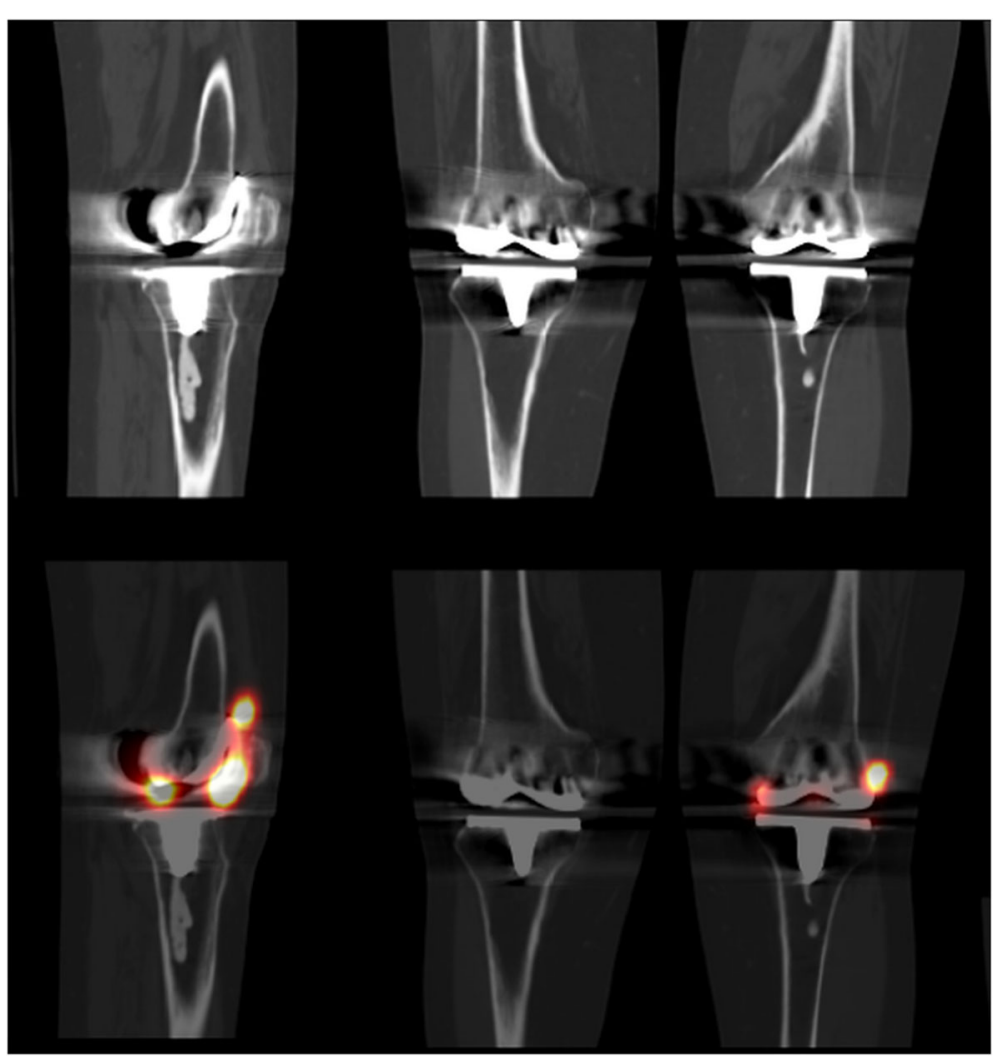

Fig. 2 Normal left knee total arthroplasty. Sagittal (left) and coronal (right) imaging with CT (top row) and fused SPECT/CT (bottom row) images showing normal tracer activity within the joint space
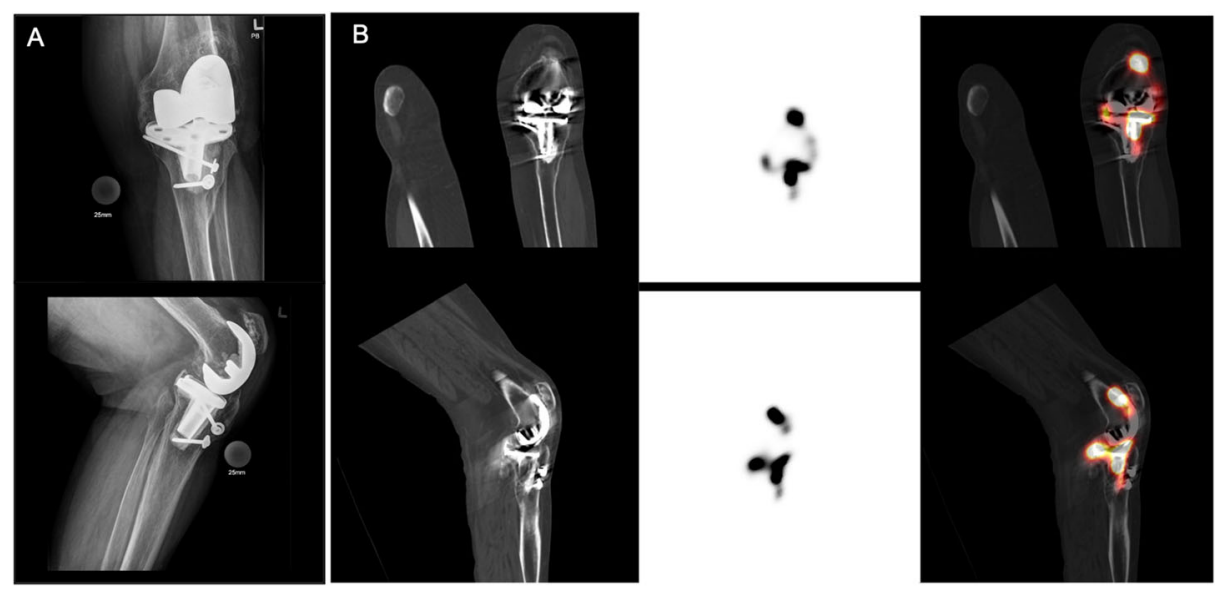

Fig. 3 Tibial component loosening of a left knee total arthroplasty. The radiograph images demonstrate no evidence of loosening (first column). Coronal (top) and sagittal CT (second column), SPECT (third column), and fused SPECT/CT (fourth column) images showing abnormal tracer activity along the bone-prosthetic interface of the tibial component 

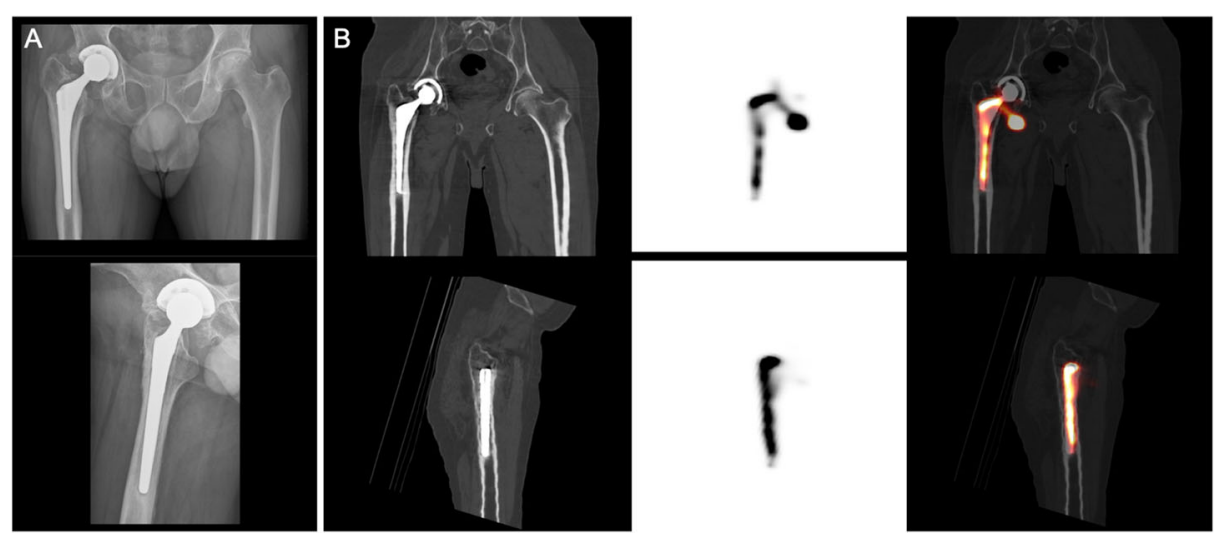

Fig. 4 Femoral component loosening of a right hip total arthroplasty. The radiograph images demonstrate lucency along the metal-bone interface (first column). Coronal (top) and sagittal CT (second column), SPECT (third column), and fused SPECT/CT (fourth column) images showing abnormal tracer activity along the bone-prosthetic interface of the femoral component

period without revision, or if the patients were subsequently diagnosed with an alternative condition that explained their symptoms. The orthopedic surgeons were aware of the patient's clinical history and not blinded to the SPECT/CT results during the follow-up period.

\section{Statistical analysis}

Age of participants and time from primary arthroplasty were reported as mean \pm standard deviation. Sex, anatomical location, and type of prosthesis were reported as ratios. The agreement between the SPECT/CT report and final diagnosis was calculated to determine the sensitivity, specificity, positive predictive values, negative predictive values, and diagnostic accuracy.

\section{Results}

The baseline demographic information and type of prostheses, as well as the average time interval from primary arthroplasty procedure to SPECT/CT imaging is reported in Table 1. There were no documented adverse reactions to the SPECT/CT arthrogram procedure.

\section{Reference standard-surgery}

In 14 of the 63 patients, revision surgery was performed following the SPECT/CT arthrogram study. Nine were knee arthroplasties and five were hip arthroplasties. For

Table 1 Baseline demographic, timing of imaging, and type of prostheses in participants

\begin{tabular}{ll}
\hline Characteristic & Participants $(\boldsymbol{n}=\mathbf{6 3})$ \\
\hline Age (years) & $69 \pm 10$ \\
Sex (M to F) & $26: 37$ \\
Anatomy (hip to knee) & $27: 36$ \\
Time from primary arthroplasty to imaging (months) & $58 \pm 51$ \\
Type of prostheses (cemented to non-cemented) & $20: 43$ \\
\hline
\end{tabular}


six of these patients, surgery was prompted by a positive SPECT/CT arthrogram study. A diagnosis of aseptic loosening was confirmed intraoperatively in all six patients. Surgery was also performed in eight patients with negative SPECT/CT arthrogram studies based on a high clinical suspicion despite negative imaging. In seven of these patients, the operative evaluation revealed an alternative non-loosening diagnosis. In one patient, the orthopedic surgeon diagnosed a subsiding tibial tray (false-negative SPECT/CT arthrogram study).

\section{Reference standard-clinical follow-up}

In 49 patients, surgery was not performed. These patients were instead followed clinically with radiologic evaluation for a minimum period of 1 -year post symptom onset. This group included 48 negative SPECT/CT arthrogram studies (true negative (TN)) and one positive SPECT/CT arthrogram study (false positive (FP)). Of the TN studies, 16 patients had pain which spontaneously resolved on follow-up and 32 patients had alternative diagnosis made by their orthopedic surgeon. Alternative diagnoses included trochanteric bursitis, spinal stenosis, anasarca, traumatic fracture, abscess, lumbar osteoarthritis, hematoma, referred arthritic pain, and patellofemoral pain syndrome. These patients had no further radiologic indications of loosening on their 1-year follow-up radiography. The one FP SPECT/CT arthrogram study was reported as positive for knee arthroplasty loosening; however, the pain resolved spontaneously without intervention. There was no evidence of loosening on follow-up radiography.

\section{Overall accuracy}

Overall, this study involving 63 SPECT/CT arthrogram evaluation of clinically suspected aseptic loosening of hip and knee arthroplasty demonstrated 6 true-positive (TP), $55 \mathrm{TN}, 1 \mathrm{FP}$, and 1 false-negative (FN) results. This results in a sensitivity of 6/7 (86\%), specificity of 55/56 (98\%), positive predictive value (PPV) of 6/7 (86\%) and negative predictive value (NPV) of 55/56 (98\%), and diagnostic accuracy of 61/63 (97\%).

\section{Discussion}

Our data confirms that SPECT/CT arthrography has a high diagnostic accuracy for detecting aseptic loosening in patients with persistent pain following primary hip and knee arthroplasties (sensitivity of $86 \%$, specificity of $98 \%$, PPV of $86 \%$, NPV of $98 \%$, and overall accuracy of 97\%). Comparable results have been reported in previously published studies. In a previous retrospective study of a smaller series of 38 SPECT/CT hip and knee arthrograms from our institution (different patient cohort than included in this study), this technique was found to have a sensitivity of $100.0 \%$, specificity of 96.0\%, PPV of $92.9 \%$, NPV of $100.0 \%$, and overall accuracy of $97.4 \%$ for loosening (Abele et al. 2015). In another study by Murer et al., examining SPECT/CT for the evaluation of loosening of total knee arthroplasties, a sensitivity of $96 \%$, specificity of $100 \%$, PPV of $72.7 \%$, and NPV of $100 \%$ was reported for detecting tibial component loosening. As well, a sensitivity of $95 \%$, specificity of $100 \%$, PPV of $42.9 \%$, and NPV of $100 \%$ was reported for detecting femoral component loosening (Murer et al. 2020). The study concluded that SPECT/CT helped in diagnosing aseptic loosening in patients with persistent pain after primary total knee arthroplasty. 
While reported studies have consistently shown high sensitivity and specificity for this technique, our evaluation of 63 patients did demonstrate $1 \mathrm{FP}$ and $1 \mathrm{FN}$ case. In the FN case, while the SPECT/CT was reported as negative for loosening, the revision surgery performed 4 months after the SPECT/CT scan demonstrated a subsiding tibial tray. The report in this case did describe substantial streak artifact on the associated CT study precluding assessment of prosthetic/osseous structures (metal artifact suppression not applied). This artifact may have contributed to the false-negative result and decreased sensitivity. With metal artifact suppression techniques now available for SPECT/CT scanners, image quality may be improved, potentially reducing the impact of this artifact. In the FP case, a review of the images demonstrated mis-registration between the SPECT and CT acquisition, likely related to patient motion. With the images re-aligned, activity is seen extending into the bone-prosthetic interface of the medial condyle region; however, this does not follow the expected contour of this interface suggesting it is likely artifact.

To diagnose loosening, several imaging modalities have been described including plain radiography, bone scintigraphy, planar nuclear arthrography, SPECT/CT, 18F-FDG-PET, and/or MRI (Barnsley and Barnsley 2019). A meta-analysis by Temmerman et al. reported a pooled sensitivity and specificity for plain radiography of $82 \%$ and $81 \%$, respectively (Temmerman et al. 2005). The most recent study on bone scintigraphy by Claassen et al. reported a sensitivity of $76 \%$ and a specificity of $83 \%$ (Claassen et al. 2014). Interestingly, planar nuclear arthrography on its own has a low reported sensitivity and specificity of 87\% and 64\% (Temmerman et al. 2005). Comparatively, we found a much higher sensitivity and specificity with SPECT/CT arthrography demonstrating the importance of the hybrid nuclear medicine imaging technique in the evaluation of aseptic loosening.

Our study has multiple limitations. First of all, as a retrospective study design, it is inherently prone to selection bias. Moreover, as the surgeons were not blinded to the SPECT/CT reports, the decision to perform the surgical revision reference standard was influenced by the outcome of the SPECT/CT diagnostic test therefore confounded by verification bias. As well, since the study involves clinical reports from multiple different readers, there is also the potential for variability in interpretation of the scans. Additionally, our study did not compare SPECT/CT arthrography results with that of SPECT/CT arthrography combined with bone scintigraphy. Further study examining both modalities could take place. Finally, while our results demonstrate a high diagnostic accuracy for our SPECT/CT arthrogram technique, it is uncertain if this technique is optimal. Specifically, a wait time of $30 \mathrm{~min}$ with ambulation or $60 \mathrm{~min}$ without ambulation may be too short. It is uncertain if a longer wait time may have impacted our FN result. There are no reported studies examining the optimal time from injection to imaging. Further study is warranted in this regard.

In summary, SPECT/CT arthrography appears to be highly accurate for the diagnosis of aseptic loosening in patients with hip or knee arthroplasties. Given this, SPECT/CT arthrography should be considered an important diagnostic adjunct, particularly for patients where there is clinical uncertainty. 
Code availability

Not applicable.

\section{Authors' contributions}

All authors contributed to the study conception and design. Material preparation, data collection, and analysis were performed by Bo Bao, Crystal Liu, Edward Masson, and Jonathan Abele. The first draft of the manuscript was written by Bo Bao and all authors commented on previous versions of the manuscript. All authors read and approved the final manuscript.

\section{Funding}

The authors did not receive financial support from any organization.

\section{Availability of data and materials}

The datasets used during the current study are available from the corresponding author on request.

\section{Ethics approval and consent to participate}

Ethical approval was granted by the local Ethics Committee of University of Alberta and all the procedures being performed were part of the routine care. Consent to participate is not applicable.

\section{Consent for publication}

Not applicable.

\section{Competing interests}

The authors declare no competing interests.

\section{Author details}

${ }^{1}$ Department of Radiology and Diagnostic Imaging, University of Alberta, 2A2.41 Walter C Mackenzie Health Sciences Centre, Edmonton, Alberta T6G 2B7, Canada. ${ }^{2}$ Cumming School of Medicine, University of Calgary, Calgary, Alberta, Canada. ${ }^{3}$ Division of Orthopedic Surgery, University Alberta, Edmonton, Alberta, Canada.

Received: 8 December 2020 Accepted: 8 February 2021

Published online: 28 February 2021

\section{References}

Abele JT, Swami VG, Russell G, Masson EC, Flemming JP (2015) The accuracy of single photon emission computed tomography/ computed tomography arthrography in evaluating aseptic loosening of hip and knee prostheses. J Arthroplast 30(9):1647-1651

Abu-Amer Y, Darwech I, Clohisy JC (2007) Aseptic loosening of total joint replacements: mechanisms underlying osteolysis and potential therapies. Arthritis Res Ther 9(Suppl 1):S6

Barnsley L, Barnsley L (2019) Detection of aseptic loosening in total knee replacements: a systematic review and metaanalysis. Skelet Radiol 48(10):1565-1572

Becker R, Bonnin M, Hofmann S (2011) The painful knee after total knee arthroplasty. Knee Surg Sports Traumatol Arthrosc 19(9):1409-1410

Claassen L, Ettinger M, Plaass C, Daniilidis K, Calliess T, Ezechieli M (2014) Diagnostic value of bone scintigraphy for aseptic loosening after total knee arthroplasty. Technol Health Care 22(5):767-773

Ethgen O, Bruyere O, Richy F, Dardennes C, Reginster JY (2004) Health-related quality of life in total hip and total knee arthroplasty. A qualitative and systematic review of the literature. J Bone Joint Surg Am 86(5):963-974

Katz JN, Earp BE, Gomoll AH (2010) Surgical management of osteoarthritis. Arthritis Care Res 62(9):1220-1228

Lohmann CH, Rampal S, Lohrengel M, Singh G (2017) Imaging in peri-prosthetic assessment: an orthopaedic perspective. EFORT Open Rev 2(5):117-125

Maradit Kremers H, Larson DR, Crowson CS, Kremers WK, Washington RE, Steiner CA et al (2015) Prevalence of total hip and knee replacement in the united states. J Bone Joint Surg Am 97(17):1386-1397

Murer AM, Hirschmann MT, Amsler F, Rasch H, Huegli RW (2020) Bone SPECT/CT has excellent sensitivity and specificity for diagnosis of loosening and patellofemoral problems after total knee arthroplasty. Knee Surg Sports Traumatol Arthrosc. 28(4):1029-1035.

Piscitelli P, Iolascon G, Innocenti M, Civinini R, Rubinacci A, Muratore M et al (2013) Painful prosthesis: approaching the patient with persistent pain following total hip and knee arthroplasty. Clin Cases Miner Bone Metab 10(2):97-110

Sharkey PF, Lichstein PM, Shen C, Tokarski AT, Parvizi J (2014) Why are total knee arthroplasties failing today--has anything changed after 10 years? J Arthroplast 29(9):1774-1778

Singh JA, Yu S, Chen L, Cleveland JD (2019) Rates of total joint replacement in the United States: future projections to 20202040 using the national inpatient sample. J Rheumatol jrheum. 46(9):1134-1140.

Sundfeldt M, Carlsson LV, Johansson CB, Thomsen P, Gretzer C (2006) Aseptic loosening, not only a question of wear: a review of different theories. Acta Orthop 77(2):177-197

Temmerman OP, Raijmakers PG, Berkhof J, Hoekstra OS, Teule GJ, Heyligers IC (2005) Accuracy of diagnostic imaging techniques in the diagnosis of aseptic loosening of the femoral component of a hip prosthesis: a meta-analysis. J Bone Joint Surg Br 87(6):781-785

Wylde V, Hewlett S, Learmonth ID, Dieppe P (2011) Persistent pain after joint replacement: prevalence, sensory qualities, and postoperative determinants. Pain 152(3):566-572

\section{Publisher's Note}

Springer Nature remains neutral with regard to jurisdictional claims in published maps and institutional affiliations. 